\title{
Boundary Value Problems for a Class of Sequential Integrodifferential Equations of Fractional Order
}

\author{
Bashir Ahmad ${ }^{1}$ and Juan J. Nieto ${ }^{1,2}$ \\ ${ }^{1}$ Department of Mathematics, Faculty of Science, King Abdulaziz University, P.O. Box 80203, Jeddah 21589, Saudi Arabia \\ ${ }^{2}$ Departamento de Análisis Matemático, Facultad de Matemáticas, Universidad de Santiago de Compostela, \\ 15782 Santiago de Compostela, Spain
}

Correspondence should be addressed to Juan J. Nieto; juanjose.nieto.roig@usc.es

Received 16 January 2013; Accepted 13 March 2013

Academic Editor: Jose Luis Sanchez

Copyright (C) 2013 B. Ahmad and J. J. Nieto. This is an open access article distributed under the Creative Commons Attribution License, which permits unrestricted use, distribution, and reproduction in any medium, provided the original work is properly cited.

We investigate the existence of solutions for a sequential integrodifferential equation of fractional order with some boundary conditions. The existence results are established by means of some standard tools of fixed point theory. An illustrative example is also presented.

\section{Introduction}

Nonlinear boundary value problems of fractional differential equations have received a considerable attention in the last few decades. One can easily find a variety of results ranging from theoretical analysis to asymptotic behavior and numerical methods for fractional equations in the literature on the topic. The interest in the subject has been mainly due to the extensive applications of fractional calculus in the mathematical modeling of several real-world phenomena occurring in physical and technical sciences; see, for example, [1-4]. An important feature of a fractional order differential operator, distinguishing it from an integer-order differential operator, is that it is nonlocal in nature. It means that the future state of a dynamical system or process based on a fractional operator depends on its current state as well as its past states. Thus, differential equations of arbitrary order are capable of describing memory and hereditary properties of some important and useful materials and processes. This feature has fascinated many researchers, and they have shifted their focus to fractional order models from the classical integerorder models. For some recent work on the topic, we refer, for instance, to [5-9]. Recently, in [10], the authors studied sequential fractional differential equations with three-point boundary conditions.
In this paper, we consider a nonlinear Dirichlet boundary value problem of sequential fractional integrodifferential equations given by

$$
\begin{gathered}
\left({ }^{c} D^{\alpha}+k^{c} D^{\alpha-1}\right) u(t)=p f(t, u(t))+q I^{\beta} g(t, u(t)), \\
0<t<1, \\
u(0)=0, \quad u(1)=0,
\end{gathered}
$$

where ${ }^{c} D^{\alpha}$ denotes the Caputo fractional derivative of order $\alpha, 1<\alpha \leq 2, I^{\beta}(\cdot)$ denotes Riemann-Liouville integral with $0<\beta<1, f, g$ are given continuous functions, $k \neq 0$, and $p, q$ are real constants. We also study the fractional integrodifferential equation (1) subject to the following boundary conditions:

$$
\begin{array}{cc}
u^{\prime}(0)+k u(0)=a, \quad u(1)=b, & a, b \in \mathbb{R}, \\
u(0)=a, & u^{\prime}(0)=u^{\prime}(1), \quad a \in \mathbb{R} .
\end{array}
$$

\section{Linear Fractional Differential Equations}

For $\alpha \in(1,2]$, we consider the following linear fractional differential equation:

$$
\left({ }^{c} D^{\alpha}+k^{c} D^{\alpha-1}\right) u(t)=h(t),
$$


where ${ }^{c} D^{\alpha}$ denotes the Caputo fractional derivative of order $\alpha$. Rewriting (1) as ${ }^{c} D^{\alpha}\left(u(t)+k^{c} D^{-1} u(t)\right)=h(t)$, we can write its solution as

$$
u(t)+k^{c} D^{-1} u(t)=\frac{1}{\Gamma(\alpha)} \int_{0}^{t}(t-s)^{\alpha-1} h(s) d s+c_{0}+c_{1} t,
$$

where $c_{0}, c_{1}$ are arbitrary constants. Now, (6) can be expressed as

$$
\begin{aligned}
u(t)= & -k \int_{0}^{t} u(s) d s+\frac{1}{\Gamma(\alpha)} \int_{0}^{t}(t-s)^{\alpha-1} h(s) d s \\
& +c_{0}+c_{1} t .
\end{aligned}
$$

Differentiating (7), we obtain

$$
u^{\prime}(t)=-k u(t)+\frac{1}{\Gamma(\alpha-1)} \int_{0}^{t}(t-s)^{\alpha-2} h(s) d s+c_{1},
$$

which can alternatively be written as

$$
\left(u(t) e^{k t}\right)^{\prime}=e^{k t}\left(\frac{1}{\Gamma(\alpha-1)} \int_{0}^{t}(t-s)^{\alpha-2} h(s) d s+c_{1}\right) .
$$

Integrating from 0 to $t$, we have

$$
u(t)=A e^{-k t}+\int_{0}^{t} e^{-k(t-s)} I^{\alpha-1} h(s) d s+B,
$$

where $A$ and $B$ are arbitrary constants, and

$$
I^{\alpha-1} h(t)=\int_{0}^{t} \frac{(t-x)^{\alpha-2}}{\Gamma(\alpha-1)} h(x) d x .
$$

Lemma 1. The unique solution of the linear equation (5) subject to the Dirichlet boundary conditions (2) is given by

$$
\begin{aligned}
u(t)= & \frac{\left(1-e^{-k t}\right)}{\left(e^{-k}-1\right)} \int_{0}^{1} e^{-k(1-s)}\left(\int_{0}^{s} \frac{(s-x)^{\alpha-2}}{\Gamma(\alpha-1)} h(x) d x\right) d s \\
& +\int_{0}^{t} e^{-k(t-s)}\left(\int_{0}^{s} \frac{(s-x)^{\alpha-2}}{\Gamma(\alpha-1)} h(x) d x\right) d s .
\end{aligned}
$$

Proof. Observe that the general solution of (5) is given by (10). Using the given boundary conditions in (10), we find that

$$
A=-B=\frac{1}{\left(1-e^{-k}\right)} \int_{0}^{1} e^{-k(1-s)}\left(\int_{0}^{s} \frac{(s-x)^{\alpha-2}}{\Gamma(\alpha-1)} h(x) d x\right) d s .
$$

Substituting the values of $A$ and $B$ in (10) yields the solution (12). This completes the proof.

In the next two lemmas, we present the unique solutions of (5) with different kinds of boundary conditions. We do not provide the proofs for these lemmas as they are similar to that of Lemma 1.
Lemma 2. The unique solution of the problem (5)-(3) is given by

$$
\begin{aligned}
u(t)= & e^{k(1-t)} \\
& \times\left[\frac{(b k-a)}{k}-\int_{0}^{1} e^{-k(1-s)}\left(\int_{0}^{s} \frac{(s-x)^{\alpha-2}}{\Gamma(\alpha-1)} h(x) d x\right) d s\right] \\
& +\int_{0}^{t} e^{-k(t-s)}\left(\int_{0}^{s} \frac{(s-x)^{\alpha-2}}{\Gamma(\alpha-1)} h(x) d x\right) d s+\frac{a}{k}
\end{aligned}
$$

Lemma 3. The unique solution of (5) with the boundary conditions (4) is

$$
\begin{aligned}
u(t)= & -\frac{\left(1-e^{-k t}\right)}{k\left(1-e^{-k}\right)} \\
& \times\left[k \int_{0}^{1} e^{-k(1-s)}\left(\int_{0}^{s} \frac{(s-x)^{\alpha-2}}{\Gamma(\alpha-1)} h(x) d x\right) d s\right. \\
& \left.\quad-\int_{0}^{1} \frac{(1-s)^{\alpha-2}}{\Gamma(\alpha-1)} h(s) d s\right] \\
+ & \int_{0}^{t} e^{-k(t-s)}\left(\int_{0}^{s} \frac{(s-x)^{\alpha-2}}{\Gamma(\alpha-1)} h(x) d x\right) d s+a .
\end{aligned}
$$

\section{Existence Results for the Nonlinear Problems}

Let $\mathscr{P}=C([0,1], \mathbb{R})$ denote the Banach space of all continuous functions from $[0,1]$ into $\mathbb{R}$ endowed with the usual norm defined by $\|x\|=\sup \{|x(t)|, t \in[0,1]\}$.

In view of Lemma 1, we transform problem (1)-(2) to an equivalent fixed point problem as

$$
u=\mathscr{V} u
$$

where $\mathscr{V}: \mathscr{P} \rightarrow \mathscr{P}$ is defined by

$(\mathscr{V} u)(t)$

$$
\begin{gathered}
=\frac{\left(1-e^{-k t}\right)}{\left(e^{-k}-1\right)} \int_{0}^{1} e^{-k(1-s)}\left(p \int_{0}^{s} \frac{(s-x)^{\alpha-2}}{\Gamma(\alpha-1)} f(x, u(x)) d x\right. \\
+q \int_{0}^{s} \frac{(s-x)^{\alpha+\beta-2}}{\Gamma(\alpha+\beta-1)} \\
\times g(x, u(x)) d x) d s \\
+\int_{0}^{t} e^{-k(t-s)}\left(p \int_{0}^{s} \frac{(s-x)^{\alpha-2}}{\Gamma(\alpha-1)} f(x, u(x)) d x\right. \\
+q \int_{0}^{s} \frac{(s-x)^{\alpha+\beta-2}}{\Gamma(\alpha+\beta-1)} \\
\times g(x, u(x)) d x) d s .
\end{gathered}
$$


In a similar manner, we can define a fixed point operator $\mathscr{V}_{1}: \mathscr{P} \rightarrow \mathscr{P}$ for the nonlinear problem (1)-(3) as follows:

$$
\begin{aligned}
& \left(\mathscr{V}_{1} u\right)(t) \\
& =e^{k(1-t)}\left[\frac{(b k-a)}{k}-\int_{0}^{1} e^{-k(1-s)}\right. \\
& \times\left(p \int_{0}^{s} \frac{(s-x)^{\alpha-2}}{\Gamma(\alpha-1)} f(x, u(x)) d x\right. \\
& +q \int_{0}^{s} \frac{(s-x)^{\alpha+\beta-2}}{\Gamma(\alpha+\beta-1)} \\
& \times g(x, u(x)) d x) d s] \\
& +\int_{0}^{t} e^{-k(t-s)}\left(p \int_{0}^{s} \frac{(s-x)^{\alpha-2}}{\Gamma(\alpha-1)} f(x, u(x)) d x\right. \\
& +q \int_{0}^{s} \frac{(s-x)^{\alpha+\beta-2}}{\Gamma(\alpha+\beta-1)} \\
& \times g(x, u(x)) d x) d s+\frac{a}{k} .
\end{aligned}
$$

A fixed point operator $\mathscr{V}_{2}: \mathscr{P} \rightarrow \mathscr{P}$ for the nonlinear problem (1)-(4) is defined by

$$
\begin{gathered}
\left(\mathscr{V}_{2} u\right)(t) \\
=-\frac{\left(1-e^{-k t}\right)}{k\left(1-e^{-k}\right)} \\
\times\left[k \int _ { 0 } ^ { 1 } e ^ { - k ( 1 - s ) } \left(p \int_{0}^{s} \frac{(s-x)^{\alpha-2}}{\Gamma(\alpha-1)}\right.\right. \\
\times f(x, u(x)) d x \\
+q \int_{0}^{s} \frac{(s-x)^{\alpha+\beta-2}}{\Gamma(\alpha+\beta-1)} \\
\quad-p \int_{0}^{1} \frac{(1-s)^{\alpha-2}}{\Gamma(\alpha-1)} f(s, u(s)) d s \\
\left.+q \int_{0}^{1} \frac{(1-s)^{\alpha+\beta-2}}{\Gamma(\alpha+\beta-1)} g(s, u(s)) d s\right] \\
+k(t-s)\left(p \int_{0}^{s} \frac{(s-x)^{\alpha-2}}{\Gamma(\alpha-1)} f(x, u(x)) d x\right. \\
+q \int_{0}^{s} \frac{(s-x)^{\alpha+\beta-2}}{\Gamma(\alpha+\beta-1)} \\
+g(x, u(x)) d x) d s+a .
\end{gathered}
$$

We only present the existence results for the problem (1)(2). Observe that problem (1)-(2) has solutions if the operator equation (16) has fixed points.

For computational convenience, we introduce the following constant:

$$
Q=\frac{2\left|1-e^{-k}\right|[|p| \Gamma(\alpha+\beta)+|q| \Gamma(\alpha)]}{|k| \Gamma(\alpha+\beta) \Gamma(\alpha)} .
$$

Theorem 4. Assume that $f, g:[0,1] \times \mathbb{R} \rightarrow \mathbb{R}$ are continuous functions satisfying the following condition:

$$
\begin{array}{r}
\left(A_{1}\right) \quad|f(t, u)-f(t, v)| \leq L_{1}|u-v|, \\
|g(t, u)-g(t, v)| \leq L_{2}|u-v|, \quad \forall t \in[0,1], \\
L_{1}, L_{2}>0, u, v \in \mathbb{R} .
\end{array}
$$

Then, the boundary value problem (1)-(2) has a unique solution if $L<1 / Q$, where $L=\max \left\{L_{1}, L_{2}\right\}$ and $Q$ is given by (20).

Proof. Let us define $M=\max \left\{M_{1}, M_{2}\right\}$, where $M_{1}, M_{2}$ are finite numbers given by $\sup _{t \in[0,1]}|f(t, 0)|=M_{1}$, $\sup _{t \in[0,1]}|g(t, 0)|=M_{2}$. Selecting $r \geq(Q M) /(1-L Q)$, we show that $\mathscr{V} B_{r} \subset B_{r}$, where $B_{r}=\{u \in \mathscr{P}:\|u\| \leq r\}$. For $u \in B_{r}$, we have

$$
\begin{gathered}
\|(\mathscr{V} u)\| \leq \sup _{t \in[0,1]}\left\{\left|\frac{1-e^{-k t}}{1-e^{-k}}\right|\right. \\
\times \int_{0}^{1} e^{-k(1-s)}\left(|p| \int_{0}^{s} \frac{(s-x)^{\alpha-2}}{\Gamma(\alpha-1)}\right. \\
\times|f(x, u(x))| d x \\
+|q| \int_{0}^{s} \frac{(s-x)^{\alpha+\beta-2}}{\Gamma(\alpha+\beta-1)} \\
\left.+\int_{0}^{t} e^{-k(t-s)} \times|g(x, u(x))| d x\right) d s \\
\times\left(|p| \int_{0}^{s} \frac{(s-x)^{\alpha-2}}{\Gamma(\alpha-1)}\right. \\
\times|f(x, u(x))| d x \\
+|q| \int_{0}^{s} \frac{(s-x)^{\alpha+\beta-2}}{\Gamma(\alpha+\beta-1)} \\
\times|g(x, u(x))| d x) d s\}
\end{gathered}
$$




$$
\begin{aligned}
& \leq \sup _{t \in[0,1]}\left\{\left|\frac{1-e^{-k t}}{1-e^{-k}}\right| \int_{0}^{1} e^{-k(1-s)}\right. \\
& \times\left(|p| \int_{0}^{s} \frac{(s-x)^{\alpha-2}}{\Gamma(\alpha-1)}\right. \\
& \times(|f(x, u(x))-f(x, 0)| \\
& +|f(x, 0)|) d x \\
& +|q| \int_{0}^{s} \frac{(s-x)^{\alpha+\beta-2}}{\Gamma(\alpha+\beta-1)} \\
& \times(|g(x, u(x))-g(x, 0)| \\
& +|g(x, 0)|) d x) d s \\
& +\int_{0}^{t} e^{-k(t-s)} \\
& \times\left(|p| \int_{0}^{s} \frac{(s-x)^{\alpha-2}}{\Gamma(\alpha-1)}\right. \\
& \times(|f(x, u(x))-f(x, 0)| \\
& +|f(x, 0)|) d x \\
& +|q| \int_{0}^{s} \frac{(s-x)^{\alpha+\beta-2}}{\Gamma(\alpha+\beta-1)} \\
& \times(|g(x, u(x))-g(x, 0)| \\
& +|g(x, 0)|) d x) d s\} \\
& \leq|p|\left(L_{1} r+M_{1}\right) \\
& \times \sup _{t \in[0,1]}\left\{\left|\frac{1-e^{-k t}}{1-e^{-k}}\right|\right. \\
& \times \int_{0}^{1} e^{-k(1-s)}\left(\int_{0}^{s} \frac{(s-x)^{\alpha-2}}{\Gamma(\alpha-1)} d x\right) d s \\
& \left.+\int_{0}^{t} e^{-k(t-s)}\left(\int_{0}^{s} \frac{(s-x)^{\alpha-2}}{\Gamma(\alpha-1)} d x\right) d s\right\} \\
& +|q|\left(L_{2} r+M_{2}\right) \\
& \times \sup _{t \in[0,1]}\left\{\left|\frac{1-e^{-k t}}{1-e^{-k}}\right|\right. \\
& \times \int_{0}^{1} e^{-k(1-s)}\left(\int_{0}^{s} \frac{(s-x)^{\alpha+\beta-2}}{\Gamma(\alpha+\beta-1)} d x\right) d s \\
& +\int_{0}^{t} e^{-k(t-s)} \\
& \left.\times\left(\int_{0}^{s} \frac{(s-x)^{\alpha+\beta-2}}{\Gamma(\alpha+\beta-1)} d x\right) d s\right\}
\end{aligned}
$$

which means that $\mathscr{V} B_{r} \subset B_{r}$.

Now, for $u, v \in \mathscr{P}$, we obtain

$$
\begin{aligned}
& \|\mathscr{V} u-\mathscr{V} v\| \\
& \leq \sup _{t \in[0,1]}\left\{\left|\frac{1-e^{-k t}}{1-e^{-k}}\right|\right. \\
& \times \int_{0}^{1} e^{-k(1-s)}\left(|p| \int_{0}^{s} \frac{(s-x)^{\alpha-2}}{\Gamma(\alpha-1)}\right. \\
& \times \mid f(s, u(s)) \\
& -f(s, v(s)) \mid d x \\
& +|q| \int_{0}^{s} \frac{(s-x)^{\alpha+\beta-2}}{\Gamma(\alpha+\beta-1)} \\
& \times \mid g(s, u(s)) \\
& -g(s, v(s)) \mid d x) d s \\
& +\int_{0}^{t} e^{-k(t-s)} \\
& \times\left(|p| \int_{0}^{s} \frac{(s-x)^{\alpha-2}}{\Gamma(\alpha-1)}\right. \\
& \times|f(s, u(s))-f(s, v(s))| d x \\
& +|q| \int_{0}^{s} \frac{(s-x)^{\alpha+\beta-2}}{\Gamma(\alpha+\beta-1)} \\
& \times|g(s, u(s))-g(s, v(s))| d x) d s\} \\
& \leq L \frac{2\left|1-e^{-k}\right|[|p| \Gamma(\alpha+\beta)+|q| \Gamma(\alpha)]}{|k| \Gamma(\alpha+\beta) \Gamma(\alpha)}\|u-v\| \\
& =L Q\|u-v\| \text {. }
\end{aligned}
$$

By the given assumption, $L<1 / Q, \mathscr{V}$ is a contraction. Thus, the conclusion of the theorem follows by the contraction mapping principle (Banach fixed point theorem).

Our next existence result relies on Krasnoselskii's fixed point theorem.

Lemma 5 (Krasnoselskii, see [11]). Let $M$ be a closed, convex, bounded, and nonempty subset of a Banach space $X$. Let $A, B$ be the operators such that (i) $A x+B y \in M$ whenever $x, y \in M$, (ii) $A$ is compact, and continuous, and (iii) $B$ is a contraction mapping. Then, there exists $z \in M$ such that $z=A z+B z$. 
Theorem 6. Let $f, g:[0,1] \times \mathbb{R} \rightarrow \mathbb{R}$ be continuous functions satisfying assumption $\left(A_{1}\right)$, and

$$
\begin{gathered}
\left(A_{2}\right) \quad|f(t, u)| \leq \mu_{1}(t), \quad|g(t, u)| \leq \mu_{2}(t), \\
\forall(t, u) \in[0,1] \times \mathbb{R}, \mu_{1}, \mu_{2} \in C\left([0,1], \mathbb{R}^{+}\right) .
\end{gathered}
$$

Then, the problem (1)-(2) has at least one solution on $[0,1]$ provided that

$$
\frac{\left|1-e^{-k}\right|\left[|p|\left\|\mu_{1}\right\| \Gamma(\alpha+\beta)+|q|\left\|\mu_{2}\right\| \Gamma(\alpha)\right]}{|k| \Gamma(\alpha+\beta) \Gamma(\alpha)}<1,
$$

where $\sup _{t \in[0,1]}\left|\mu_{i}(t)\right|=\left\|\mu_{i}\right\|, i=1,2$.

Proof. Let us fix

$$
\bar{r} \geq \frac{2\left|1-e^{-k}\right|\left[|p|\left\|\mu_{1}\right\| \Gamma(\alpha+\beta)+|q|\left\|\mu_{2}\right\| \Gamma(\alpha)\right]}{|k| \Gamma(\alpha+\beta) \Gamma(\alpha)}
$$

and consider $B_{\bar{r}}=\{u \in \mathscr{P}:\|u\| \leq \bar{r}\}$. We define the operators $\psi_{1}$ and $\psi_{2}$ on $B_{\bar{r}}$ as

$$
\begin{aligned}
& \left(\psi_{1} u\right)(t)=\int_{0}^{t} e^{-k(t-s)}\left(p \int_{0}^{s} \frac{(s-x)^{\alpha-2}}{\Gamma(\alpha-1)} f(x, u(x)) d x\right. \\
& +q \int_{0}^{s} \frac{(s-x)^{\alpha+\beta-2}}{\Gamma(\alpha+\beta-1)} \\
& \times g(x, u(x)) d x) d s, \\
& t \in[0,1], \\
& \left(\psi_{2} u\right)(t)=\frac{\left(1-e^{-k t}\right)}{\left(e^{-k}-1\right)} \\
& \times \int_{0}^{1} e^{-k(1-s)}\left(p \int_{0}^{s} \frac{(s-x)^{\alpha-2}}{\Gamma(\alpha-1)} f(x, u(x)) d x\right. \\
& +q \int_{0}^{s} \frac{(s-x)^{\alpha+\beta-2}}{\Gamma(\alpha+\beta-1)} \\
& \times g(x, u(x)) d x) d s, \\
& t \in[0,1] \text {. }
\end{aligned}
$$

For $u, v \in B_{\bar{r}}$, we find that

$$
\begin{aligned}
& \left\|\psi_{1} u+\psi_{2} v\right\| \\
& \quad \leq \frac{2\left|1-e^{-k}\right|\left[|p|\left\|\mu_{1}\right\| \Gamma(\alpha+\beta)+|q|\left\|\mu_{2}\right\| \Gamma(\alpha)\right]}{|k| \Gamma(\alpha+\beta) \Gamma(\alpha)} \\
& \leq \bar{r} .
\end{aligned}
$$

Thus, $\psi_{1} u+\psi_{2} v \in B_{\bar{r}}$. It follows from assumption $\left(A_{1}\right)$ together with $(25)$ that $\psi_{2}$ is a contraction mapping.
Continuities of $f$ and $g$ imply that the operator $\psi_{1}$ is continuous. Also, $\psi_{1}$ is uniformly bounded on $B_{\bar{r}}$ as

$\left\|\psi_{1} u\right\|$

$$
\leq \frac{\left|1-e^{-k}\right|\left[|p|\left\|\mu_{1}\right\| \Gamma(\alpha+\beta)+|q|\left\|\mu_{2}\right\| \Gamma(\alpha)\right]}{|k| \Gamma(\alpha+\beta) \Gamma(\alpha)} .
$$

Now, we prove the compactness of the operator $\psi_{1}$. In view of $\left(A_{1}\right)$, we define

$$
\sup _{(t, u) \in[0,1] \times B_{\bar{r}}}|f(t, u)|=\bar{f}, \quad \sup _{(t, u) \in[0,1] \times B_{\bar{r}}}|g(t, u)|=\bar{g} .
$$

Consequently, we have

$$
\begin{aligned}
& \left\|\left(\psi_{1} u\right)\left(t_{2}\right)-\left(\psi_{1} u\right)\left(t_{1}\right)\right\| \\
& \leq\left|e^{-k t_{2}}-e^{-k t_{1}}\right| \int_{0}^{t_{1}} e^{k s}\left(|p| \bar{f} \int_{0}^{s} \frac{(s-x)^{\alpha-2}}{\Gamma(\alpha-1)} d x\right. \\
& \left.\quad+|q| \bar{g} \int_{0}^{s} \frac{(s-x)^{\alpha+\beta-2}}{\Gamma(\alpha+\beta-1)} d x\right) d s \\
& \leq \int_{t_{1}}^{t_{2}} e^{-k\left(t_{2}-s\right)} \\
& \quad \times\left(|p| \bar{f} \int_{0}^{s} \frac{(s-x)^{\alpha-2}}{\Gamma(\alpha-1)} d x+|q| \bar{g} \int_{0}^{s} \frac{(s-x)^{\alpha+\beta-2}}{\Gamma(\alpha+\beta-1)} d x\right) d s
\end{aligned}
$$

which is independent of $u$ and tends to zero as $t_{2} \rightarrow t_{1}$. Thus, $\psi_{1}$ is relatively compact on $B_{\bar{r}}$. Hence, by the Arzelá-Ascoli theorem, $\psi_{1}$ is compact on $B_{\bar{r}}$. Thus, all the assumptions of Lemma 5 are satisfied. So, by the conclusion of Lemma 5 , problem (1)-(2) has at least one solution on $[0,1]$.

Now, we show the existence of solutions for the problem (1)-(2) via Leray-Schauder alternative.

Lemma 7 (nonlinear alternative for single valued maps, see [12]). Let $E$ be a Banach space, $C$ a closed, convex subset of $E, U$ an open subset of $C$, and $0 \in U$. Suppose that $F: \bar{U} \rightarrow C$ is a continuous, compact (that is, $F(\bar{U})$ is a relatively compact subset of C) map. Then, either

(i) F has a fixed point in $\bar{U}$, or

(ii) there is a $u \in \partial U$ (the boundary of $U$ in $C$ ) and $\lambda \epsilon$ $(0,1)$ with $u=\lambda F(u)$.

Theorem 8. Let $f, g:[0,1] \times \mathbb{R} \rightarrow \mathbb{R}$ be continuous functions and the following assumptions hold.

$\left(A_{3}\right)$ There exist functions $\sigma_{1}, \sigma_{2} \in C\left([0,1], \mathbb{R}^{+}\right)$, and nondecreasing functions $\psi_{1}, \psi_{2}: \mathbb{R}^{+} \rightarrow \mathbb{R}^{+}$ such that $|f(t, u)| \leq \sigma_{1}(t) \psi_{1}(\|u\|),|g(t, u)| \leq$ $\sigma_{2}(t) \psi_{2}(\|u\|)$, for all $(t, u) \in[0,1] \times \mathbb{R}$. 
$\left(A_{4}\right)$ There exists a constant $M>0$ such that

$$
\begin{gathered}
M \times\left(\left(2 | 1 - e ^ { - k } | \left[|p| \psi_{1}(\|u\|) \Gamma(\alpha+\beta)\left\|\sigma_{1}\right\|\right.\right.\right. \\
\left.\left.+|q| \psi_{2}(\|u\|)\left\|\sigma_{2}\right\| \Gamma(\alpha)\right]\right) \\
\left.\times(|k| \Gamma(\alpha+\beta) \Gamma(\alpha))^{-1}\right)^{-1}>1 .
\end{gathered}
$$

Then, the boundary value problem (1)-(2) has at least one solution on $[0,1]$.

Proof. Consider the operator $\mathscr{V}: \mathscr{P} \rightarrow \mathscr{P}$ with $u=\mathscr{V} u$, where

$$
\begin{aligned}
& (\mathscr{V} u)(t)=\frac{\left(1-e^{-k t}\right)}{\left(e^{-k}-1\right)} \\
& \times \int_{0}^{1} e^{-k(1-s)}\left(p \int_{0}^{s} \frac{(s-x)^{\alpha-2}}{\Gamma(\alpha-1)}\right. \\
& \times f(x, u(x)) d x \\
& +q \int_{0}^{s} \frac{(s-x)^{\alpha+\beta-2}}{\Gamma(\alpha+\beta-1)} \\
& \times g(x, u(x)) d x) d s \\
& +\int_{0}^{t} e^{-k(t-s)}\left(p \int_{0}^{s} \frac{(s-x)^{\alpha-2}}{\Gamma(\alpha-1)} f(x, u(x)) d x\right. \\
& +q \int_{0}^{s} \frac{(s-x)^{\alpha+\beta-2}}{\Gamma(\alpha+\beta-1)} \\
& \times g(x, u(x)) d x) d s
\end{aligned}
$$

We show that $\mathscr{V}$ maps bounded sets into bounded sets in $C([0,1], \mathbb{R})$. For a positive number $r$, let $B_{r}=\{u \in$ $C([0,1], \mathbb{R}):\|u\| \leq r\}$ be a bounded set in $C([0,1], \mathbb{R})$. Then, $|(\mathscr{V} u)(t)|$

$$
\begin{aligned}
& \leq\left|\frac{1-e^{-k t}}{1-e^{-k}}\right| \int_{0}^{1} e^{-k(1-s)} \\
& \times\left(|p| \int_{0}^{s} \frac{(s-x)^{\alpha-2}}{\Gamma(\alpha-1)}|f(x, u(x))| d x\right. \\
& \left.+|q| \int_{0}^{s} \frac{(s-x)^{\alpha+\beta-2}}{\Gamma(\alpha+\beta-1)}|g(x, u(x))| d x\right) d s \\
& +\int_{0}^{t} e^{-k(t-s)}\left(|p| \int_{0}^{s} \frac{(s-x)^{\alpha-2}}{\Gamma(\alpha-1)}|f(x, u(x))| d x\right. \\
& +|q| \int_{0}^{s} \frac{(s-x)^{\alpha+\beta-2}}{\Gamma(\alpha+\beta-1)} \\
& \times|g(x, u(x))| d x) d s
\end{aligned}
$$

$$
\begin{aligned}
& \leq\left|\frac{1-e^{-k t}}{1-e^{-k}}\right| \\
& \times \int_{0}^{1} e^{-k(1-s)}\left(|p| \int_{0}^{s} \frac{(s-x)^{\alpha-2}}{\Gamma(\alpha-1)} \sigma_{1}(x) \psi_{1}(\|u\|) d x\right. \\
& \left.+|q| \int_{0}^{s} \frac{(s-x)^{\alpha+\beta-2}}{\Gamma(\alpha+\beta-1)} \sigma_{2}(x) \psi_{2}(\|u\|) d x\right) d s \\
& +\int_{0}^{t} e^{-k(t-s)}\left(|p| \int_{0}^{s} \frac{(s-x)^{\alpha-2}}{\Gamma(\alpha-1)} \sigma_{1}(x) \psi_{1}(\|u\|) d x\right. \\
& +|q| \int_{0}^{s} \frac{(s-x)^{\alpha+\beta-2}}{\Gamma(\alpha+\beta-1)} \\
& \left.\times \sigma_{2}(x) \psi_{2}(\|u\|) d x\right) d s \\
& \leq|p| \psi_{1}(r)\left\|\sigma_{1}\right\| \\
& \times\left[\left|\frac{1-e^{-k t}}{1-e^{-k}}\right| \int_{0}^{1} e^{-k(1-s)}\left(\int_{0}^{s} \frac{(s-x)^{\alpha-2}}{\Gamma(\alpha-1)} d x\right) d s\right. \\
& \left.+\int_{0}^{t} e^{-k(t-s)}\left(\int_{0}^{s} \frac{(s-x)^{\alpha-2}}{\Gamma(\alpha-1)} d x\right) d s\right] \\
& +|q|\left\|\sigma_{2}\right\| \psi_{2}(r)\left[\left|\frac{1-e^{-k t}}{1-e^{-k}}\right|\right. \\
& \times \int_{0}^{1} e^{-k(1-s)}\left(\int_{0}^{s} \frac{(s-x)^{\alpha+\beta-2}}{\Gamma(\alpha+\beta-1)} d x\right) d s \\
& \left.+\int_{0}^{t} e^{-k(t-s)}\left(\int_{0}^{s} \frac{(s-x)^{\alpha+\beta-2}}{\Gamma(\alpha+\beta-1)} d x\right) d s\right] \\
& \leq\left(2 | 1 - e ^ { - k } | \left[|p| \psi_{1}(\|u\|) \Gamma(\alpha+\beta)\left\|\sigma_{1}\right\|\right.\right. \\
& \left.\left.+|q| \psi_{2}(\|u\|)\left\|\sigma_{2}\right\| \Gamma(\alpha)\right]\right) \\
& \times(|k| \Gamma(\alpha+\beta) \Gamma(\alpha))^{-1} \text {. }
\end{aligned}
$$

Consequently,

$$
\begin{aligned}
\|\mathscr{V} x\| \leq & \left(2\left|1-e^{k}\right|\right. \\
& \times\left[|p| \psi_{1}(\|u\|) \Gamma(\alpha+\beta)\left\|\sigma_{1}\right\|\right. \\
& \left.\left.\quad+|q| \psi_{2}(\|u\|)\left\|\sigma_{2}\right\| \Gamma(\alpha)\right]\right) \\
& \times(|k| \Gamma(\alpha+\beta) \Gamma(\alpha))^{-1} .
\end{aligned}
$$

Next, we show that $\mathscr{V}$ maps bounded sets into equicontinuous sets of $C([0,1], \mathbb{R})$. Let $t_{1}, t_{2} \in[0,1]$ with $t_{1}<t_{2}$ and 
$u \in B_{r}$, where $B_{r}$ is a bounded set of $C([0,1], \mathbb{R})$. Then, we obtain

$$
\begin{aligned}
& \left\|(\mathscr{V} u)\left(t_{2}\right)-(\mathscr{V} u)\left(t_{1}\right)\right\| \\
& \leq\left|\frac{-e^{-k t_{2}}+e^{-k t_{1}}}{1-e^{-k}}\right| \\
& \times \int_{0}^{1} e^{-k(1-s)}\left(|p| \psi_{1}(r)\left\|\sigma_{1}\right\|\right. \\
& \times \int_{0}^{s} \frac{(s-x)^{\alpha-2}}{\Gamma(\alpha-1)} d x \\
& +|q| \psi_{2}(r)\left\|\sigma_{2}\right\| \\
& \left.\times \int_{0}^{s} \frac{(s-x)^{\alpha+\beta-2}}{\Gamma(\alpha+\beta-1)} d x\right) d s \\
& +\left|e^{-k t_{2}}-e^{-k t_{1}}\right| \\
& \times \int_{0}^{t_{1}} e^{k s}\left(|p| \psi_{1}(r)\left\|\sigma_{1}\right\|\right. \\
& \times \int_{0}^{s} \frac{(s-x)^{\alpha-2}}{\Gamma(\alpha-1)} d x \\
& +|q| \psi_{2}(r) \| \sigma_{2}|| \\
& \left.\times \int_{0}^{s} \frac{(s-x)^{\alpha+\beta-2}}{\Gamma(\alpha+\beta-1)} d x\right) d s \\
& +\int_{t_{1}}^{t_{2}} e^{-k\left(t_{2}-s\right)}\left(|p| \psi_{1}(r)\left\|\sigma_{1}\right\| \int_{0}^{s} \frac{(s-x)^{\alpha-2}}{\Gamma(\alpha-1)} d x\right. \\
& +|q| \psi_{2}(r)\left\|\sigma_{2}\right\| \\
& \left.\times \int_{0}^{s} \frac{(s-x)^{\alpha+\beta-2}}{\Gamma(\alpha+\beta-1)} d x\right) d s
\end{aligned}
$$

Obviously, the right hand side of the previous inequality tends to zero independently of $u \in B_{r}$ as $t_{2}-t_{1} \rightarrow 0$. As $\mathscr{V}$ satisfies the previous assumptions, therefore it follows by the Arzelá-Ascoli theorem that $\mathscr{V}: C([0,1], \mathbb{R}) \rightarrow C([0,1], \mathbb{R})$ is completely continuous.

The proof will be complete by the application of the LeraySchauder nonlinear alternative (Lemma 7) once we establish the boundedness of the set of all solutions to equations $u=$ $\lambda \mathscr{V} u$ for $\lambda \in(0,1)$.
Let $u$ be a solution. Then, for $t \in[0,1]$, and using the computations in proving that $\mathscr{V}$ is bounded, we have

$$
\begin{aligned}
|u(t)|= & |\lambda(\mathscr{V} u)(t)| \\
\leq & \left(2\left|1-e^{-k}\right|\right. \\
& \times\left[|p| \psi_{1}(\|u\|) \Gamma(\alpha+\beta)\left\|\sigma_{1}\right\|\right. \\
& \left.\left.\quad+|q| \psi_{2}(\|u\|)\left\|\sigma_{2}\right\| \Gamma(\alpha)\right]\right) \\
& \times(|k| \Gamma(\alpha+\beta) \Gamma(\alpha))^{-1} .
\end{aligned}
$$

Consequently, we have

$$
\begin{gathered}
\|u\| \times\left(\left(2 \mid 1-e^{-k \mid}\left[|p| \psi_{1}(\|u\|) \Gamma(\alpha+\beta)\left\|\sigma_{1}\right\|\right.\right.\right. \\
\left.\left.+|q| \psi_{2}(\|u\|)\left\|\sigma_{2}\right\| \Gamma(\alpha)\right]\right) \\
\left.\times(|k| \Gamma(\alpha+\beta) \Gamma(\alpha))^{-1}\right)^{-1} \leq 1 .
\end{gathered}
$$

In view of $\left(A_{4}\right)$, there exists $M$ such that $\|u\| \neq M$. Let us set

$$
U=\{u \in C([0,1], \mathbb{R}):\|u\|<M\} .
$$

Note that the operator $\mathscr{V}: \bar{U} \rightarrow C([0,1], \mathbb{R})$ is continuous and completely continuous. From the choice of $U$, there is no $u \in \partial U$ such that $u=\lambda \mathscr{V}(u)$ for some $\lambda \in(0,1)$. Consequently, by the nonlinear alternative of Leray-Schauder type (Lemma 7), we deduce that $\mathscr{V}$ has a fixed point $u \in \bar{U}$ which is a solution of the problem (1)-(2). This completes the proof.

Example 9. Consider a boundary value problem of integrodifferential equations of fractional order given by

$$
\begin{gathered}
\left({ }^{c} D^{3 / 2}+2{ }^{c} D^{1 / 2}\right) u(t) \\
=\frac{1}{2} f(t, u(t))+I^{1 / 2} g(t, u(t)), \quad 0<t<1, \\
u(0)=0, \quad u(1)=0,
\end{gathered}
$$

where $\alpha=3 / 2, k=2, p=1 / 2, q=1, \beta=1 / 2, f(t, u)=$ $(|u|(2+|u|)) /(3(1+|u|))+4 t, g(t, u)=(1 / 4) \tan ^{-1} u+\cos ^{2} t+t^{3}+$ 5. With the given data, it is found that $L_{1}=2 / 3, L_{2}=1 / 4$ as $|f(t, u)-f(t, v)| \leq(2 / 3)|u-v|,|g(t, u)-g(t, v)| \leq(1 / 4)|u-v|$, and

$$
Q=\frac{2\left|1-e^{-k}\right|[|p| \Gamma(\alpha+\beta)+|q| \Gamma(\alpha)]}{|k| \Gamma(\alpha+\beta) \Gamma(\alpha)} \simeq 1.3525 .
$$

Clearly, $L=\max \left\{L_{1}, L_{2}\right\}=2 / 3$ and $L<1 / Q$. Thus, all the assumptions of Theorem 4 are satisfied. Hence, by the conclusion of Theorem 4, the problem (40) has a unique solution.

\section{Acknowledgment}

The authors thank the anonymous referees for their valuable comments. The research of J. J. Nieto has been partially 
supported by Ministerio de Economia y Competitividad (Spain), project MTM2010-15314, and co-financed by the European Community fund FEDER.

\section{References}

[1] S. G. Samko, A. A. Kilbas, and O. I. Marichev, Fractional Integrals and Derivatives: Theory and Applications, Gordon and Breach Science Publishers, Yverdon, Switzerland, 1993.

[2] I. Podlubny, Fractional Differential Equations, vol. 198 of Mathematics in Science and Engineering, Academic Press, San Diego, Calif, USA, 1999.

[3] A. A. Kilbas, H. M. Srivastava, and J. J. Trujillo, Theory and Applications of Fractional Differential Equations, vol. 204 of North-Holland Mathematics Studies, Elsevier Science B.V., Amsterdam, The Netherlands, 2006.

[4] D. Baleanu, K. Diethelm, E. Scalas, and J. J. Trujillo, Fractional Calculus Models and Numerical Methods, vol. 3 of Series on Complexity, Nonlinearity and Chaos, World Scientific Publishing, Boston, Mass, USA, 2012.

[5] D. Băleanu, O. G. Mustafa, and R. P. Agarwal, "On $L^{p}$-solutions for a class of sequential fractional differential equations," Applied Mathematics and Computation, vol. 218, no. 5, pp. 20742081, 2011.

[6] R. P. Agarwal and B. Ahmad, "Existence theory for anti-periodic boundary value problems of fractional differential equations and inclusions," Computers \& Mathematics with Applications, vol. 62, no. 3, pp. 1200-1214, 2011.

[7] B. Ahmad and J. J. Nieto, "Riemann-Liouville fractional integrodifferential equations with fractional nonlocal integral boundary conditions," Boundary Value Problems, vol. 2011, article 36, 9 pages, 2011.

[8] N. J. Ford and M. L. Morgado, "Fractional boundary value problems: analysis and numerical methods," Fractional Calculus and Applied Analysis, vol. 14, no. 4, pp. 554-567, 2011.

[9] A. Aghajani, Y. Jalilian, and J. J. Trujillo, "On the existence of solutions of fractional integro-differential equations," Fractional Calculus and Applied Analysis, vol. 15, no. 1, pp. 44-69, 2012.

[10] B. Ahmad and J. J. Nieto, "Sequential fractional differential equations with three-point boundary conditions," Computers \& Mathematics with Applications, vol. 64, no. 10, pp. 3046-3052, 2012.

[11] D. R. Smart, Fixed Point Theorems, Cambridge University Press, London, UK, 1980.

[12] A. Granas and J. Dugundji, Fixed Point Theory, Springer Monographs in Mathematics, Springer, New York, NY, USA, 2003. 


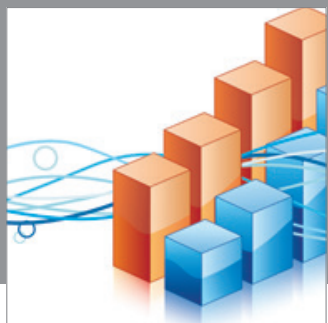

Advances in

Operations Research

mansans

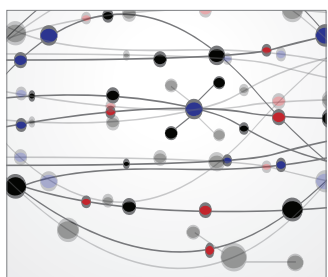

The Scientific World Journal
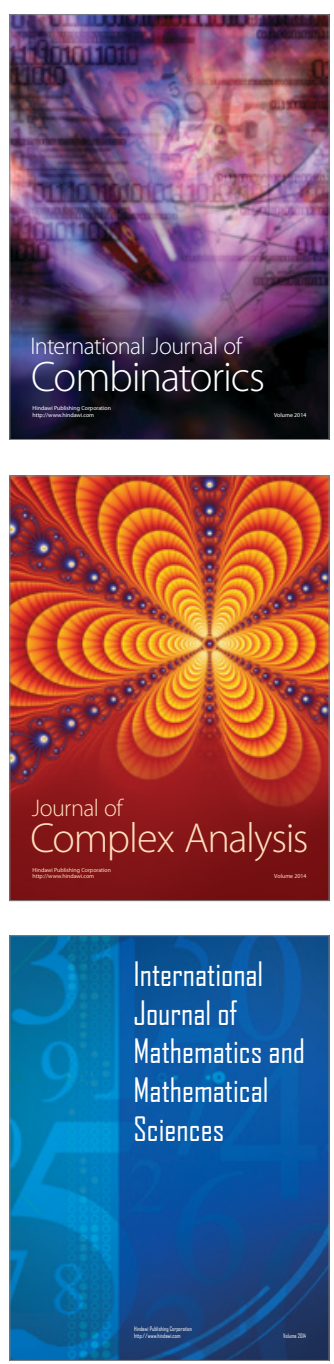
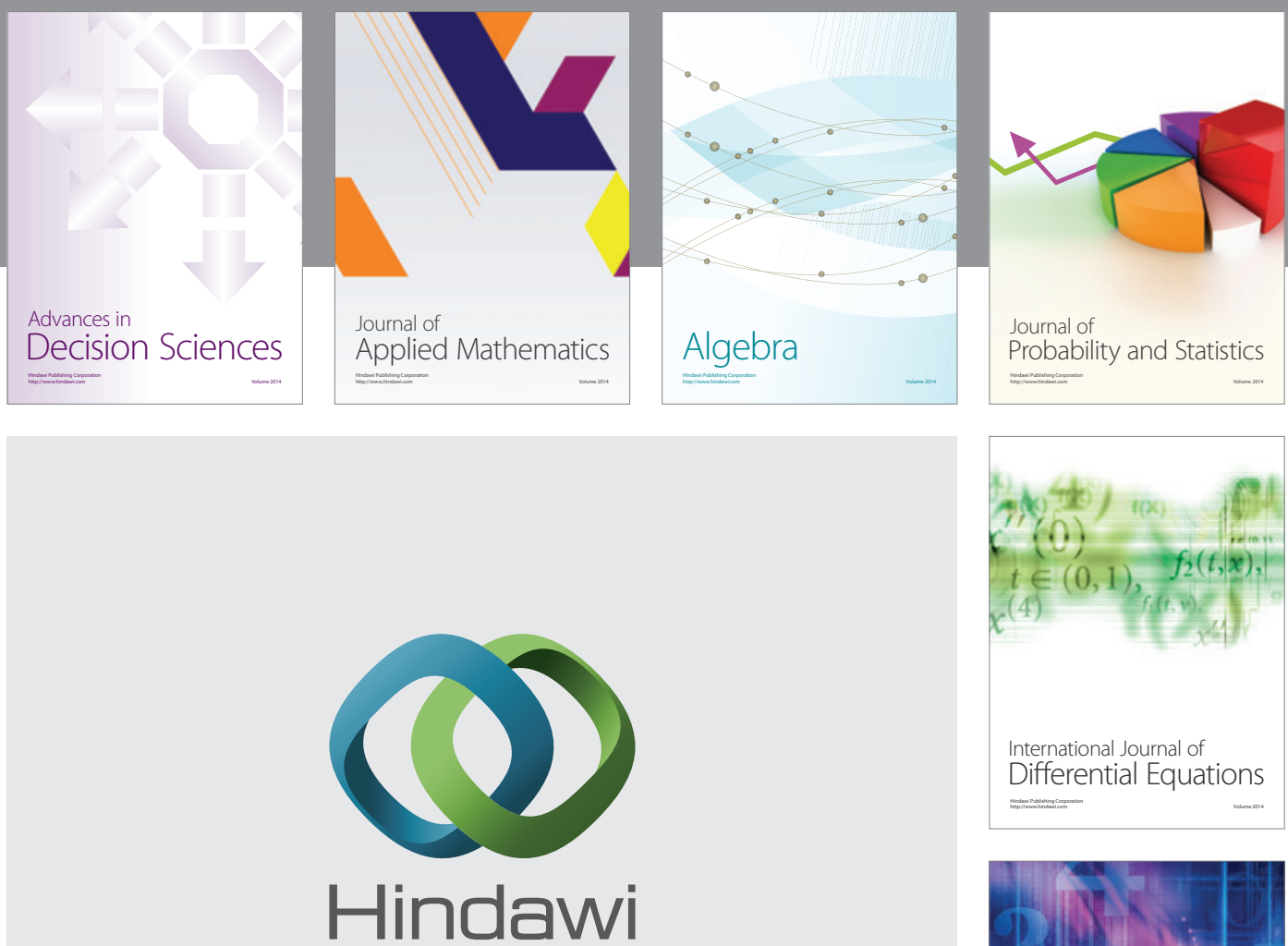

Submit your manuscripts at http://www.hindawi.com
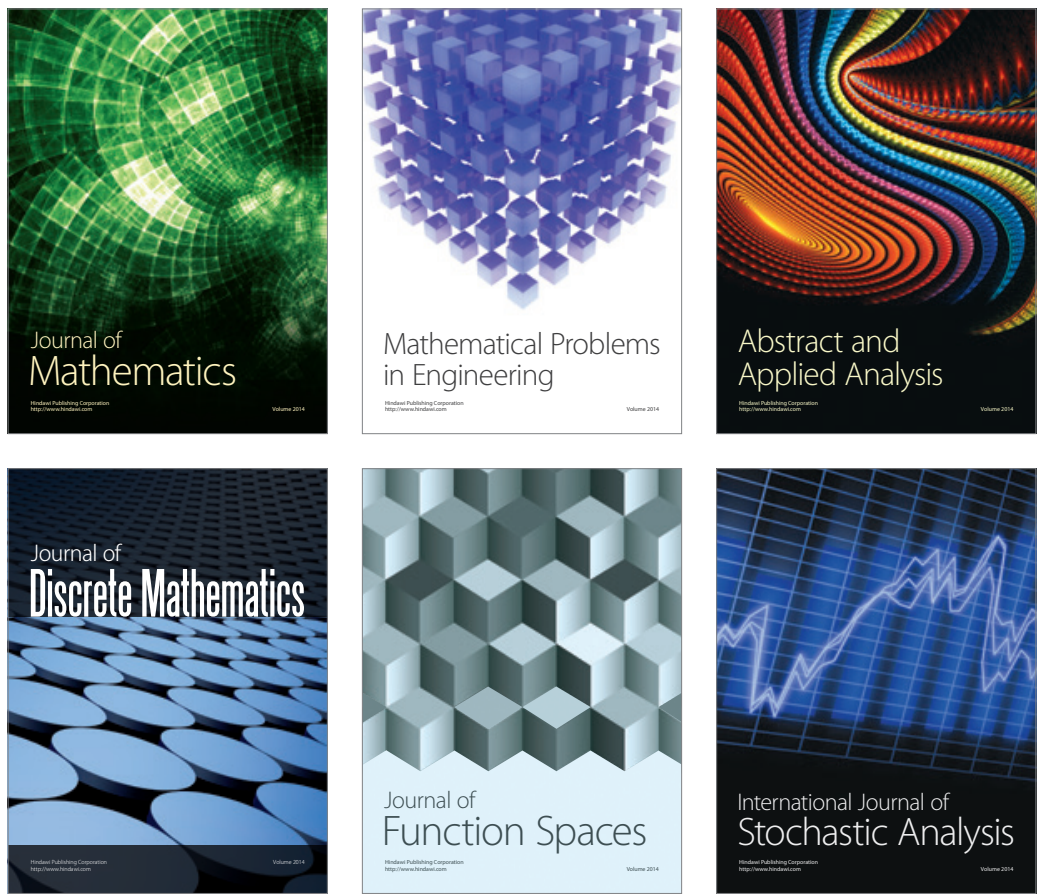

Journal of

Function Spaces

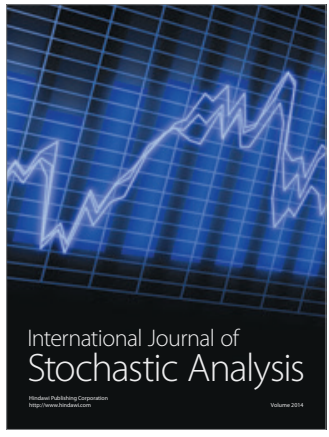

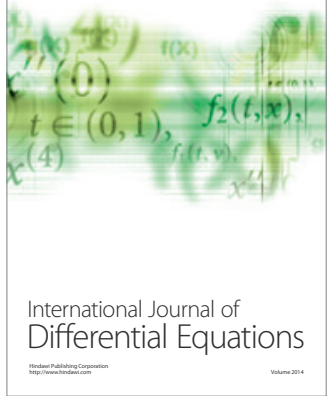
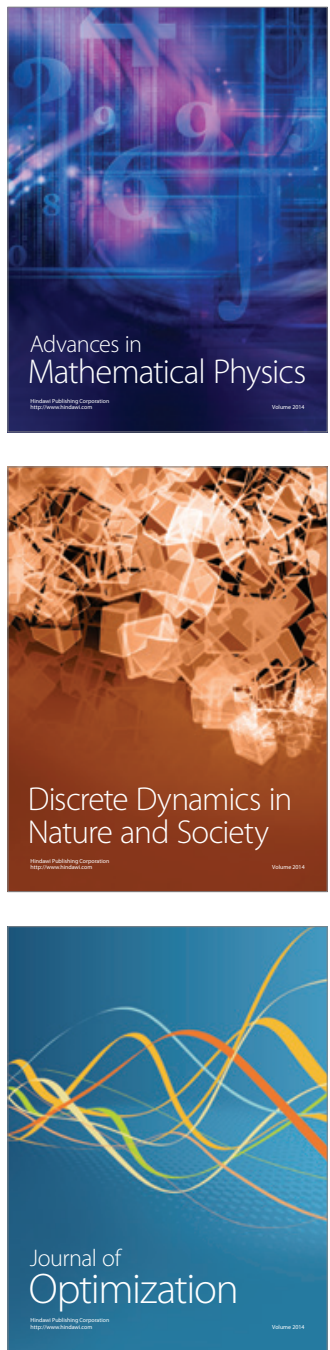